\title{
The Effectiveness of Mindfulness-Based Stress Reduction Group Therapy in Emotion Regulation Among Multiple Sclerosis Patients
}

\author{
Hanieh Nobakht (iD) ${ }^{1}$, Sana Nourimoghadam (iD ${ }^{1,}{ }^{*}$ and Zahra Nikmanesh (iD) ${ }^{1}$ \\ ${ }^{1}$ Department of Psychology, Faculty of Education and Psychology, University of Sistan and Baluchestan, Zahedan, Iran \\ "Corresponding author: Department of Psychology, Faculty of Education and Psychology, University of Sistan and Baluchestan, Zahedan, Iran. Email: \\ s.nourimoghadam@gmail.com
}

Received 2022 January 25; Accepted 2022 January 25.

\begin{abstract}
Background: In addition to physical issues, multiple sclerosis (MS) patients experience many psychological problems that make their living conditions difficult.

Objectives: This study aimed at examining the effectiveness of mindfulness-based stress reduction (MBSR) group therapy in emotion regulation among MS patients.

Methods: This quasi-experimental study followed a pre-test, post-test, and follow-up design with a control group. The statistical population included all patients with MS who were referred to Sistan and Baluchestan MS Community from December 2017 to February 2018. Among these people, 30 patients were selected as the sample using the convenience sampling method and were randomly assigned to a control group and an experimental group (each including 15 patients). Eight 60-minute sessions of MBSR therapy were conducted on the experimental group. A post-test was carried out after completing all these sessions, and a follow-up was performed a month later. The Self-Regulation Inventory developed by Ibanez et al. was used as the data collection tool. The analysis of covariance was used to analyze the obtained data.

Results: The results showed that MBSR group therapy was effective in improving emotion regulation in patients with MS in the post-test and follow-up $(\mathrm{P}<0.05)$.

Conclusions: Authorities are suggested employing MBSR group therapy to boost emotion regulation among patients with chronic diseases.
\end{abstract}

Keywords: Mindfulness, Stress, Emotions, Regulation, Multiple Sclerosis

\section{Background}

Multiple sclerosis (MS) is an autoimmune, inflammatory, chronic, and progressive disease that nerve lesions can diagnose with damaged myelin sheaths in the white matter areas of the brain, spinal cord, and optic nerves. MS is one of the most important diseases of the central nervous system, one of the most prevalent neurological diseases in humans, and the most common disease leading to disability in young people, which affects nearly 2.5 million people worldwide (1). Because this disease occurs suddenly and unpredictably, it causes a lot of stress in the affected person and affects different aspects of the person's life (2).

Variations in the psychological characteristics of people with MS, in addition to by neurological changes in the brain structure, may be due to the many challenges that MS, as a stressor, brings about for patients' physical and psychological well-being (3). Because of their difficult and frustrating conditions, MS patients deal with numerous mental disorders and diseases, among which a reduction in quality of life, difficulty in regulating emotions, anxiety, depression, sexual dysfunction, problems in playing social roles, and a reduction in the relatives' well-being can be mentioned as the most important ones (4). Therefore, it seems necessary to pay attention to the psychological wellbeing of patients with MS (5).

One of the factors affecting the well-being of people with MS is emotion regulation (6). Emotion regulation refers to strategies used to reduce, enhance, or maintain emotional experiences. This component is an intrinsic aspect of emotional response tendencies (7). Cognitive emotion regulation strategies are divided into two categories: negative and positive strategies.

Negative strategies include rumination, self-blame, blaming others, and catastrophe, and positive strategies include positive refocusing, positive reassessment, and ac- 
ceptance (8).

Due to the many problems and complications caused by drug abuse that occur for patients with chronic diseases, such as MS, and considering the need to reduce stress in these patients, it seems necessary to use nonpharmacological restraints that can reduce stress and improve restraint relief in these patients. These therapies, which are referred to as complementary therapies, have a societal nature that is used to increase patients' mental and physical well-being. Mindfulness-based stress reduction (MBSR) is one of these therapies (9).

Mindfulness-based cognitive therapy includes a part of MBSR therapy presented by Kabat-Zinn. This therapy was developed as group therapy for people who have a history of depression and are considered vulnerable (10).

There is ample evidence that MBSR programs have an effect on health (11). Practicing mindfulness skills increases the ability of MS sufferers to cope with negative emotional states and enables them to cope effectively (12). The concept of mindfulness helps them to accept negative emotions as part of their lives that are not permanent (13).

Babaei Nadinluye et al. (14) figured out that there was a direct and significant correlation between mindfulness and positive cognitive emotion regulation and a diverse relationship between negative cognitive emotion regulation and mindfulness. Norouzi and Hashemi (15) demonstrated that mindfulness-based cognitive therapy was beneficial for improving emotion regulation and psychosocial and self-care outcomes of patients with chronic diseases. Leyland et al. (16) showed that mindfulness training had positive effects and increased people's emotion regulation and attention.

In this regard, Khezri-Moghadam et al. concluded that the MBSR program is effective in reducing depression and anxiety in patients with MS (17).

Zamani et al. showed the effectiveness of MBSR on reducing anxiety, depression, and cognitive disorders in patients with MS, and these researchers suggested that attention to psychological therapies in these patients can be useful (18).

Piri-Kamrani et al. suggested that training MBSR can be a way to achieve an effective solution to reduce stress and regulate emotion and improve the mental status of patients with MS (19). Therefore, it is expected that the patient who has achieved mindfulness can also improve his physical, mental, and emotional health.

A review of research shows that the effects of stress on the health of patients with MS depend on their coping skills and emotional regulation strategies, and in thera- peutic interventions, coping strategies and emotional regulation of these patients can be targeted for treatment.

\section{Objectives}

The aim of this study was to determine the effectiveness of mindfulness training on emotion regulation in patients with MS.

\section{Methods}

This quasi-experimental study was performed on patients with MS referring to Zahedan MS Association in 2017 after receiving the permission of the Ethics Committee (Code of Ethics Committee: IR.USB.rec.97.008) of Sistan and Baluchistan University. The inclusion criteria were being diagnosed with MS by a neurologist, not suffering from any diseases other than MS according to a physician's opinion, and not undergoing any other psychological interventions. The exclusion criteria were dealing with mental and/or cognitive disorders, having a history of substance addiction, and dealing with other neurological disorders. Based on the analysis by Waller et al., G-Power was used to choose 30 individuals as the test sample, considering the variables and the highest standard deviation $\left(\mathrm{m}_{1}=11.8\right.$, $\mathrm{m}_{2}=7.2, \mathrm{sd}_{1}=\mathrm{sd}_{2}=6.4$, alpha $=0.05$, and test power of 0.80 .

For allocating the sample, is a container containing the blue and green color cards was used, and after careful study, the participants were given written consent forms and one card at random. The blue card indicated that the sample was in the intervention group (15 people), and the green card indicated the allocation to the control group (15 people).

The instruments used in the study included a questionnaire of demographic information and the Self-Regulation Inventory (SRI-25). This 25 -item inventory measures selfregulation in five areas, ie, positive actions, controllability, expression of feelings and needs, assertiveness, and wellbeing seeking, on a 5-point Likert-type scale, ranging from 1 to 5 (20). The minimum score is 25 , and the maximum score is 125. A higher score indicates a higher level of emotion regulation.

Cronbach's alpha coefficients for the Persian version of this inventory were $0.93,0.91$, and 0.92 for all of the subjects, male subjects, and female subjects, respectively, indicating a high internal consistency $(21,22)$. Cronbach's alpha coefficient in the present study was 0.87 .

Before the intervention, written consent was taken from the samples, and the questionnaires were distributed 
by the researcher among the patients and the subjects were screened considering the research variables and were divided into two identical groups according to their age and scores on emotion regulation. The contents and objectives of these sessions were extracted from a perspective proposed by Kabat-Zinn in 1990 (10). In the intervention group, eight training sessions of 90 minutes were held once a week in the form of questions and answers based on group discussion (Table 1), and in the control group, routine care was performed, and no intervention was performed. At the end of each session, some homework assignments were assigned, and at the beginning of each session, the assignments and topics discussed in the previous session were reviewed. The content and summary of each session are presented in (Table 1 ).

Data analysis was done using descriptive and inferential statistics, including mean, standard deviation, and repeated-measures ANOVA. The Shapiro-Wilk test was employed to examine the normal distribution of data in the pre-test, post-test, and follow-up. The repeated-measures ANOVA was used to investigate the research hypothesis. SPSS version 23.0 was further utilized to analyze the data.

\section{Results}

The study sample included 30 MS patients aged 23 to 49 years old. Also, $46.8 \%$ of the patients in the experimental group and $40.1 \%$ of the patients in the control group were in the age range of 23 to 31 years. Furthermore, 33.3\% of the patients in the experimental and control groups were in the age range of 32 to 39 years, and $20 \%$ of the patients in the experimental group, and $26.8 \%$ of the patients in the control group were in the age range of 40 to 49 years. Regarding educational levels, $60 \%$ of the patients in the experimental group and $13.3 \%$ of the patients in the control group did not have a diploma, $6.7 \%$ of the patients in the experimental group, and $40 \%$ of the patients in the control group had a diploma, and $33.4 \%$ of the patients in the experimental group and $46.7 \%$ of the patients in the control group had a bachelor's degree $(\mathrm{P}<0.05)$.

Leven's and Machley's tests were used to evaluate the homogeneity of variance and to investigate the sphericity hypothesis of the main variable of the research. The significance of Leven's and Machley tests in the emotion regulation variable was greater than 0.05; therefore, the hypothesis of homogeneity of variances was confirmed, and the null hypothesis for the equal variance of the scores of the two groups in all variables was confirmed.
The mean score of emotion regulation in the experimental group increased in the post-test and follow-up in comparison to the control group) $\mathrm{P} \leq 0.001$ (Table 2).

The results showed that the emotion regulation scores in the three time points were significantly different. In other words, a significant interaction was observed between the score (pre-test, post-test, and follow-up) and the group in the emotion regulation variable. These results indicated the effectiveness of MBSR group therapy in regulating emotions. Therefore, the multivariate test showed that in post-test and follow-up compared to pre-test, emotion regulation (experiment) changed significantly. Statistical power equal to $1 \%$ indicated the significant accuracy of these effects.

The results of the repeated measures ANOVA showed the significant effects of time (pre-test, post-test, and follow-up) $(\mathrm{P} \leq 0.02$ and $\mathrm{F}=5.28)$ on the time-group interaction $(P<0,0001$ and $F=61.25)$. There was a significant difference between the groups (membership in the experimental group versus the control group $)(\mathrm{P} \leq 0.02$ and $\mathrm{F}=$ 5.95) in emotion regulation (Table 3 ).

These results indicated that MBSR therapy was effective in increasing emotion regulation. In addition, the time variable did not change from post-test to follow-up. Therefore, the comparisons showed that after the test and follow-up, compared to the pre-test, emotion regulation in the experimental group increased significantly. Statistical powers of $0.60 \%$ and one also indicated significant accuracy in these causal relationships (Table 3 ).

The results of the Bonferroni test on the difference in emotion regulation between the experimental and control groups showed that there was a significant difference between the scores of individuals in the post-test and followup stages (Table 4).

\section{Discussion}

The main objective of the current study was to examine the effectiveness of the MBSR group therapy in emotion regulation among MS patients. The finding of this study showed that the MBSR group therapy, in the post-test and follow-up, improved emotion regulation in MS patients. This finding is consistent with the results of several studies (14-19).

Babaei Nadinluye et al. (14) and Norouzi and Hashemi (15) demonstrated that there was a direct and significant correlation between mindfulness and positive cognitive emotion regulation and a diverse relationship between negative cognitive emotion regulation and mindfulness. 
Nobakht Het al.

\begin{tabular}{l}
\hline Table 1. Summary of the Structure and Contents of the Therapy Sessions of the Mindfulness-Based Stress Reduction Therapy \\
\hline The Intervention Plan \\
\hline Session
\end{tabular}

\begin{tabular}{|c|c|c|c|c|}
\hline Group & No. & Pre-test & Post-test & Follow-up \\
\hline \multicolumn{5}{|l|}{$\begin{array}{l}\text { Emotion } \\
\text { regulation }\end{array}$} \\
\hline $\begin{array}{l}\text { Experimen- } \\
\text { tal }\end{array}$ & 15 & $68.26 \pm 6.40$ & $74.33 \pm 5.57$ & $75.53 \pm 5.68$ \\
\hline Control & 15 & $73.33 \pm 9.93$ & $70.33 \pm 8.96$ & $72.06 \pm 8.33$ \\
\hline
\end{tabular}

Khezri-Moghadam et al. (17), Zamani et al. (18), PiriKamrani et al. (19) reported that mindfulness training was effective in regulating emotions. In other words, mindfulness training increased emotion regulation. Mahmood et al. showed that mindfulness training increased positive mood, improved negative mood, and reduced stress, anxiety, and depression. Leyland et al. (16) demonstrated that mindfulness training had positive effects and enhanced people's emotion regulation and attention.

Accordingly, when explaining these findings, it can be said that MBSR is one of the most important factors in regulating and expressing emotions, and emotion regulation has an important role in adapting to MS. Indeed, recognizing emotions improves patients' mental health. Patients who use functional emotion regulation strategies can respond to life problems in a better way and effectively deal with the psychological distress caused by the disease. Mindfulness training enables people to live in the present, be aware of internal and external phenomena, nurture a non-judgmental attitude towards events and accept them easily, and respond to the phenomena constructively instead of employing non-constructive approaches. Therefore, they can deal with life events much better and perform better in different aspects of life. This can help people to modulate and manage pleasant and unpleasant emotions and aid them not easily being affected by negative emotions.

\section{Conclusions}

Therefore, according to what was mentioned earlier, the findings obtained from this study on the effectiveness of the MBSR group therapy can help therapists, doctors, and counselors as a convenient and understandable method, and also it aids patients to improve their conditions. It is recommended that the MBSR group therapy be commonly used for MS patients and authorities of the MS associations of hospitals and other organizations that have direct contact with this group of patients. It is also recommended to develop and equip mentoring mindfulnessbased training workshops since they are effective and constructive measures that improve these patients' well-being and emotion regulation. On the other hand, positive interventions can aid people to increase their attention to themselves and control themselves to experience low levels of stress. Successful coping with stressful factors and 
Nobakht Het al.

\begin{tabular}{|c|c|c|c|c|c|}
\hline Source & Sum of Squares & DF & Mean Squares & $\mathbf{F}$ & P-Value \\
\hline \multicolumn{6}{|l|}{ Between } \\
\hline Time & 117.60 & 1 & 117.60 & 5.28 & 0.02 \\
\hline Time \& group & 1363.26 & 1 & 1363.26 & 61.25 & 0.0001 \\
\hline Error & 623.13 & 28 & 22.25 & & \\
\hline \multicolumn{6}{|l|}{ Within } \\
\hline Group & 728.17 & 1 & 728.17 & 5.95 & 0.02 \\
\hline Error & 3424.22 & 28 & & & \\
\hline
\end{tabular}

\begin{tabular}{|c|c|c|c|c|c|}
\hline Variables & Group 1(I) & Group $2(\mathrm{~J})$ & Means Deferent & Standard Error & P-Value \\
\hline \multicolumn{6}{|c|}{ Emotion regulation } \\
\hline \multirow{2}{*}{ Post-test } & experiment & control & 5.68 & 2.33 & 0.02 \\
\hline & control & experiment & -5.68 & 2.33 & 0.02 \\
\hline \multirow{2}{*}{ Follow-up } & experiment & control & 2.80 & 1.21 & 0.02 \\
\hline & control & experiment & -2.80 & 1.21 & 0.02 \\
\hline
\end{tabular}

difficult situations can improve people's ability to face various problems.

\subsection{Limitations and Recommendations}

Limitations of the present study include using nonprobability sampling, considering a sample that was limited to a few centers, and not studying the role of demographic variables cultural and social factors.

It is suggested that similar research be conducted on a larger sample to examine the role of demographic variables and compare this approach with another approach to reduce psychological problems in these patients.

References

\section{Acknowledgments}

This article was an excerpt from the master's thesis approved by the Ethics Committee (Code of Ethics Committee: IR.USB.rec.97.008) of Sistan and Baluchistan University. The authors would like to appreciate the support of the Vice-Chancellor for Research and Technology of Sistan and Baluchistan University and all the participants in this research project.

\section{Footnotes}

Authors' Contribution: All authors discussed the results and contributed to the final manuscript.
Conflict of Interests: The authors did not declare any conflicts of interests.

Ethical Approval: This research project was part of a Master's thesis approved by Sistan and Baluchistan University with the code IR.USB.rec.97.008.

Funding/Support: No financial support was received for this research.

Informed Consent: Written informed consent was obtained from all patients.

\section{References}

1. Łabuz-Roszak B, Kubicka-Baczyk K, Pierzchała K, Horyniecki M, Machowska-Majchrzak A, Augustyńska-Mutryn D, et al. [Quality of life in multiple sclerosis-association with clinical features, fatigue and depressive syndrome]. Psychiatr Pol. 2013;47(3):433-42. Polish.

2. Segal BM. Clinical Immunology. 5th ed. London, UK: Elsevier; 2019. p. 891-902.

3. Khanjani Z. [Effective psychological factors on multiple sclerosis: personality traits, depression, anxiety and stress]. Med J Tabriz Univ Med Sci. 2012;34(4):60-7. Persian.

4. Celik DB, Poyraz EC, Bingol A, Idiman E, Ozakbas S, Kaya D. Sexual dysfunction in multiple sclerosis: gender differences. $J$ Neurol Sci. 2013;324(1-2):17-20. doi: 10.1016/j.jns.2012.08.019. [PubMed: 23079605].

5. Narimani M, Alisari NK, Mosazadeh T. The Effect of ExcitementFocused Therapy and Impulse Control Training on Educational Burnout and Positive and Negative Emotions in Students with and Without Learning Disabilities. J Learn Disabil. 2014;3(3):79-99.

6. Soleymani E, Habibi M, Tajoddini E. The Assessment of cognitive emotion regulation strategies, sensory processing sensitivity and anxi- 
ety sensitivity in patients with multiple sclerosis. J Arak Univ Med Sci. 2016;19(8):57-66.

7. Gross JJ. Handbook of emotion regulation. New York, USA: Guilford Publications; 2015.

8. Pollock NC, McCabe GA, Southard AC, Zeigler-Hill V. Pathological personality traits and emotion regulation difficulties. Pers Individ Differ. 2016;95:168-77. doi: 10.1016/j.paid.2016.02.049.

9. Leahy RL. Introduction: Emotional Schemas, Emotion Regulation, and Psychopathology. Int J Cogn Ther. 2013;5(4):359-61. doi: 10.1521/ijct.2012.5.4.359.

10. Kabat-Zinn J, Hanh TN. Full catastrophe living: Using the wisdom of your body and mind to face stress, pain, and illness. Delta; 2009.

11. Prakash RS, Whitmoyer P, Aldao A, Schirda B. Mindfulness and emotion regulation in older and young adults. Aging Ment Health. 2020;21(1):77-87. doi: 10.1080/13607863.2015.1100158. [PubMed: 26537859].

12. Veehof MM, Oskam MJ, Schreurs KMG, Bohlmeijer ET. Acceptancebased interventions for the treatment of chronic pain: a systematic review and meta-analysis. Pain. 2011;152(3):533-42. doi: 10.1016/j.pain.2010.11.002. [PubMed: 21251756].

13. Dehghani M, Esmaeilian N, Akbari F, Hassanvand M, Nikmanesh E. Evaluating the psychometric properties and factorial structure of the five dimensional mindfulness questionnaire. Thoughts Behav Clin Psychol. 2014;9(33):77-87.

14. Babaei Nadinluye K, Mikaeli Manee F, Pezeshki H, Bafande H, Abdi H. Relationship between Mindfulness and Meta-Emotion on Predicting Emotional Adjustment of Novice Nurses. Iran J Nurs. 2017;30(105):1122. doi: 10.29252/ijn.30.105.11.

15. Norouzi H, Hashemi E. The efficacy of Mindfulness-Based Cognitive
Therapy (MBCT) on emotion regulation among patients with breast cancer. Iran QJ Breast Dis. 2017;10(2):38-48.

16. Leyland A, Rowse G, Emerson LM. Experimental effects of mindfulness inductions on self-regulation: Systematic review and meta-analysis. Emotion. 2019;19(1):108-22. doi: 10.1037/emo0000425. [PubMed: 29578742].

17. Khezri-Moghadam N, Ghorbani N, Bahrami-Ehsan H. Efficacy of mindfulness-based stress reduction (MBSR) on the psychological symptoms of multiple sclerosis patients. J Res Behav Sci. 2013;10(7):597-608.

18. Zamani N, Rahmati A, Fazilatpoor M. The Effect of CognitiveBehavioral Stress Management Training on Anxiety, Depression and Cognitive Disorder of Individuals with Multiple Sclerosis. J Clin Psychol. 2014;5(4).

19. Piri-Kamrani M, Dehghan F, Bashiri H. The effectiveness of stress management skills training on perceived stress and resiliency of women with multiple sclerosis. J Health Care. 2016;17(4):319-28.

20. Marqués MJ, Ibáñez MI, Ruipérez MA, Moya J, Ortet G. The SelfRegulation Inventory (SRI): Psychometric properties of a health related coping measure. Pers Individ Differ. 2005;39(6):1043-54. doi: 10.1016/j.paid.2005.02.030.

21. Ghalehban M, Besharat MA. Examination and comparison of alexithymia and self-regulation in patients with substance abuse disorder and normal individuals. University of Tehran; 2009.

22. Ebadi N, Faghihi A. [The Effectiveness of Positive Negation Education on Increasing the Life Expectancy of Women Without Wife of Ahwaz City, Emphasizing the Quran]. Imam Khomeini J Educ Res. 2010;3(2):6174. Persian. 